\title{
Forward-Backward Asymmetries of Lepton Pairs in Events with a Large-Transverse-Momentum Jet at Hadron Colliders
}

\author{
F. del Aguila, ${ }^{1}$ Ll. Ametller, ${ }^{2}$ and P. Talavera ${ }^{3}$ \\ ${ }^{1}$ Departamento de Física Teórica y del Cosmos and Centro Andaluz de Física de Partículas Elementales (CAFPE), \\ Universidad de Granada, E-18071 Granada, Spain \\ ${ }^{2}$ Departament de Física i Enginyeria Nuclear, Universitat Politècnica de Catalunya, E-08034 Barcelona, Spain \\ ${ }^{3}$ Departament d'Estructura i Constituents de la Matèria, Universitat de Barcelona, E-08028 Barcelona, Spain
} (Received 18 June 2002; published 1 October 2002)

\begin{abstract}
We discuss forward-backward charge asymmetries for lepton-pair production in association with a large-transverse-momentum jet at hadron colliders. The lepton charge asymmetry relative to the jet direction $A_{\mathrm{FB}}^{j}$ gives a new determination of the effective weak mixing angle $\sin ^{2} \theta_{\mathrm{eff}}^{\text {lept }}\left(M_{Z}^{2}\right)$ with a statistical precision after cuts of $\sim 10^{-3}\left(8 \times 10^{-3}\right)$ at LHC (Tevatron). This is to be compared with the current uncertainty at LEP and SLD from the asymmetries alone, $2 \times 10^{-4}$. The identification of $b$ jets also allows for the measurement of the bottom-quark- $Z$ asymmetry $A_{\mathrm{FB}}^{b}$ at hadron colliders, the resulting statistical precision for $\sin ^{2} \theta_{\text {eff }}^{\text {lept }}\left(M_{Z}^{2}\right)$ being $\sim 9 \times 10^{-4}\left(2 \times 10^{-2}\right.$ at Tevatron), also lower than the reported precision at $e^{+} e^{-}$colliders, $3 \times 10^{-4}$.
\end{abstract}

DOI: $10.1103 /$ PhysRevLett.89.161802

PACS numbers: 13.85.-t, 14.70.-e

The possibility of using hadron colliders to perform precision tests of the electroweak standard model (SM) is a challenge for the Fermilab Tevatron and the CERN Large Hadron Collider (LHC) experiments [1,2]. Indeed, the large neutral gauge boson production cross section can allow for a precise determination of the effective weak mixing angle $\sin ^{2} \theta_{\text {eff }}^{\text {lept }}$, the optimum observable being the forward-backward charge asymmetry of lepton pairs $A_{\mathrm{FB}}$ in the Drell-Yan process $q \bar{q} \rightarrow \gamma, Z \rightarrow$ $l^{-} l^{+}$, with $l=e$ or $\mu[3,4]$. The Collider Detector Facility (CDF) Collaboration has reported a measurement of $A_{\mathrm{FB}}=0.070 \pm 0.016$ for $e^{-} e^{+}$pair invariant masses between 75 and $105 \mathrm{GeV}$ at the Fermilab Tevatron Run I [5]. The expected precision to be reached at Run II with an integrated luminosity of $10 \mathrm{fb}^{-1}$ has been estimated to be $\sim 0.1 \%$, corresponding to a precision for $\sin ^{2} \theta_{\text {eff }}^{\text {lept }}$ of $\sim 0.05 \%$ [6]. At LHC with an integrated luminosity of $100 \mathrm{fb}^{-1}$, the asymmetry precision will be further improved by a factor $\sim 6$ and the weak mixing angle one by a factor $\sim 3$. This is comparable to the current global fit precision, 0.00014, but, for instance, a factor $\sim 2$ better than the effective weak mixing angle precision obtained from the bottom forward-backward asymmetry at Large Electron-Positron (LEP) collider at CERN and SLAC Large Detector (SLD) at Stanford, 0.00031 [7].

The associated production of a neutral gauge boson $V=\gamma, Z$ (with $V \rightarrow l^{-} l^{+}$) and a jet has also a large cross section, especially at LHC, thus can also allow for a precise determination of the effective weak mixing angle. This next to leading order (NLO) correction to $V$ production is a genuine new process when the detection of the extra jet is required. In particular, gluons can be also initial states, and the large gluon content of the proton at high energy tends to make the $V$ and $V j$ production cross sections of similar size. A neutral gauge boson with an accompanying jet is produced at tree level by $q \bar{q}$ and $g \stackrel{(-)}{q}$ collisions, amounting the latter to $\sim 83 \%(\sim 48 \%)$ of the total $V j$ cross section at LHC, $\sqrt{s}=14 \mathrm{TeV}$ (Tevatron, $\sqrt{s}=2 \mathrm{TeV}$ ), for the cuts below. In this Letter we point out that the forward-backward charge asymmetry of the lepton pairs can be measured in this process either relative to a direction fixed by the initial state $A_{\mathrm{FB}}$ as in the Drell-Yan case, or relative to the final jet direction $A_{\mathrm{FB}}^{j}$. The former is adapted to obtain the asymmetry from the events $q \bar{q} \rightarrow V g \rightarrow l^{-} l^{+} g$, and the latter from $g \stackrel{(-)}{q} \rightarrow$ $V^{(-)} q \rightarrow l^{-} l^{+} \stackrel{(-)}{q}$. Both asymmetries give similar precision for $\sin ^{2} \theta_{\text {eff }}^{\text {lept }}$ at LHC but not at Tevatron, where $A_{\mathrm{FB}}$ gives a precision almost 1 order of magnitude higher. However, $A_{\mathrm{FB}}^{j}$ also allows for the measurement of flavor asymmetries. Thus, if we require the final jet to be a $b$ quark, we can make a new measurement of $A_{\mathrm{FB}}^{b}$. This is especially interesting given its observed deviation at the $Z$ pole from the SM prediction, $2.9 \sigma$ [7]. Although the corresponding effective weak mixing angle precision in principle expected at LHC, $8.9 \times 10^{-4}$, is lower than the one reported by LEP and SLD, $3.1 \times 10^{-4}$, it is similar to the difference between the central values resulting from $A_{\mathrm{FB}}^{b}$ at the $Z$ pole and the global fit to all data [7].

In the following, we discuss these asymmetries and estimate the expected statistical precision at LHC and Tevatron. Here we present tree level results with no detailed detector simulation, although any attempt to fit real data demands including electromagnetic and strong as well as electroweak radiative corrections [6]. Although large, these corrections, which are expected to modify the predicted asymmetries appreciably, are NLO. Particularly worrisome is a priori the production of a neutral gauge boson with two jets. The phase space region where one of them is too soft or collinear dominates the total cross section. The corresponding logarithmic behavior 
then compensates for the corresponding extra $\alpha_{s}$ suppression factor. However, this leading contribution must be included in the parton distribution functions and then subtracted from the $V j j$ cross section to avoid double counting, the resulting correction being actually NLO [8]. This process is also further enhanced at high energy for it has gluon fusion contributions. However, at LHC energies these are still smaller than the $V j$ cross section. A detailed calculation of the NLO corrections is in progress. The simulation of the experimental setup is also an essential ingredient to describe the observed asymmetries. We try to mimic the experimental conditions in our parton calculation, but a real simulation is eventually needed.

In Drell-Yan production of lepton pairs, the forwardbackward charge asymmetry has to be measured relative to the initial quark direction. In $p \bar{p}$ collisions, this is identified with the direction of the proton because it has more quarks than antiquarks.

$$
A_{\mathrm{FB}}=\frac{F-B}{F+B},
$$

with

$$
\begin{aligned}
& F=\int_{0}^{1} \frac{d \sigma}{d \cos \theta_{\mathrm{CS}}} d \cos \theta_{\mathrm{CS}}, \\
& B=\int_{-1}^{0} \frac{d \sigma}{d \cos \theta_{\mathrm{CS}}} d \cos \theta_{\mathrm{CS}}
\end{aligned}
$$

and

$$
\cos \theta_{\mathrm{CS}}=\frac{2\left(p_{z}^{l^{-}} E^{l^{+}}-p_{z}^{l^{+}} E^{l^{-}}\right)}{\sqrt{\left(p^{l^{-}}+p^{l^{+}}\right)^{2}} \sqrt{\left(p^{l^{-}}+p^{l^{+}}\right)^{2}+\left(p_{T}^{l^{-}}+p_{T}^{l^{+}}\right)^{2}}},
$$

where $\theta_{\mathrm{CS}}$ is the Collins-Soper angle [9]. The fourmomenta are measured in the laboratory frame and $p_{T}^{\mu} \equiv$ $\left(0, p_{x}, p_{y}, 0\right)$. In $p p$ colliders, the quark direction is fixed by the rapidity of the lepton pair. This implies defining $\cos \theta_{\mathrm{CS}}$ with an extra sign factor $\frac{\left|p_{z}^{l^{-}}+p_{z}^{l^{+}}\right|}{p_{z}^{l^{+}}+p_{z}^{l^{+}}}$. In $V j$ production one can use the same asymmetry $A_{\mathrm{FB}}$ or define a new one relative to the final jet, $A_{\mathrm{FB}}^{j}$. In this last case the corresponding angle $\theta$ in Eq. (2) is defined for $p p$ collisions as the angle between $l^{-}$and the direction opposite to the jet in the $l^{-} l^{+}$rest frame,

$$
\cos \theta=\frac{\left(p^{l^{-}}-p^{l^{+}}\right) \cdot p^{j}}{\left(p^{l^{-}}+p^{l^{+}}\right) \cdot p^{j}}
$$

The corresponding asymmetry which is suited to $g \stackrel{(-)}{q}$ collisions does not vanish because the proton contains many more quarks than antiquarks. However, in $p \bar{p}$ colliders there are produced as many quarks as antiquarks, and this asymmetry vanishes unless some difference is made between them. Hence, $\cos \theta$ is defined with an extra sign factor $\frac{\left|p_{z}\right|}{p_{z}}, p=p^{l^{-}}+p^{l^{+}}+p^{j}$, which corresponds 161802-2 to assume that the largest rapidity parton is an (anti)quark if it is along the (anti)proton direction. Besides, $V j$ events also allow for measuring a flavor asymmetry if the final jet is identified and its charge determined, as in the case of $V b$ production and $A_{\mathrm{FB}}^{b}$. For these events $A_{\mathrm{FB}}$ is less significant. In order to obtain $A_{\mathrm{FB}}^{b}$ in $p p$ or $p \bar{p}$ colliders, one must use $\cos \theta$ in Eq. (4) but multiplied by a $+(-)$ sign for $b$ (anti)quarks, $-\operatorname{sgn}\left(Q_{b}\right)$ with $Q_{b}$ the $b$ charge.

Let us present our numerical results for $l^{-} l^{+} j$ and $l^{-} l^{+} b$ at LHC and Tevatron in turn. We work in the effective Born approximation [2] and use the MartinRoberts-Stirling-Thorne parton distribution functions [10]. The $K$ factors for LHC and Tevatron, 1.1 and 1.2, respectively [11], are not included. Otherwise, they would slightly improve our statistical precision estimates. Besides, we count only electron pairs. For muons the main differences would be the pseudorapidity coverage $[12,13]$ and the size of the radiative corrections involving the lepton mass [6], which are not considered here anyway. A realistic simulation should include the detector acceptances and efficiencies. We imitate the experimental setup at LHC (Tevatron), smearing the lepton and jet energies using values based on the CDF specifications [14]

$$
\begin{aligned}
\frac{\Delta E^{e}}{E^{e}} & =\frac{10(20) \%}{\sqrt{E^{e}}}+0.3(2) \%, \\
\frac{\Delta E^{j}}{E^{j}} & =\frac{50(80) \%}{\sqrt{E^{j}}}+3(5) \%,
\end{aligned}
$$

with $E$ in $\mathrm{GeV}$, and requiring that the momenta $p$, pseudorapidities $\eta$, and separation in the pseudorapidityazimuthal angle plane $\Delta R$ satisfy

$$
\begin{array}{rlrl}
p_{t}^{e} & =\sqrt{p_{T}^{e} 2}>20 \mathrm{GeV}, \quad p_{t}^{j} & =\sqrt{p_{T}^{j 2}}>50(30) \mathrm{GeV}, \\
\left|\eta^{e, j}\right| & <2.5, \\
\Delta R_{e, j} & >0.4,
\end{array}
$$

respectively, unless otherwise stated. In Fig. 1(a) we plot the $p p \rightarrow V j \rightarrow e^{-} e^{+} j$ cross section, with $V=\gamma, Z$ and the cuts above for LHC, as a function of $M_{e^{-}} e^{+}=$ $\sqrt{\left(p^{e^{-}}+p^{e^{+}}\right)^{2}}$ (upper curves). The distributions with (solid line) and without (dashed line) smearing are overimposed, no difference being apparent. In Fig. 2(a) we show the corresponding charge asymmetries, $A_{\mathrm{FB}}$ relative to the initial parton and $A_{\mathrm{FB}}^{j}$ to the final jet. Both give similar results, although the former is adapted to the $q \bar{q}$ collisions and the latter to the $g \stackrel{(-)}{q}$ ones. We do not include hadronization neither detector simulation which, as the smearing, mainly affect the asymmetries, in particular, due to the fact that the directions of the jets are related but not equal to the directions of the parent partons. In the figures we also show the $p p \rightarrow V \stackrel{(-)}{b} \rightarrow e^{-} e^{+} \stackrel{(-)}{b}$ cross section, assuming a $b$-tagging efficiency of 50\% [12], and the corresponding asymmetry $A_{\mathrm{FB}}^{b}$, assuming no charge misassignment (thick lines). The cross section is

161802-2 

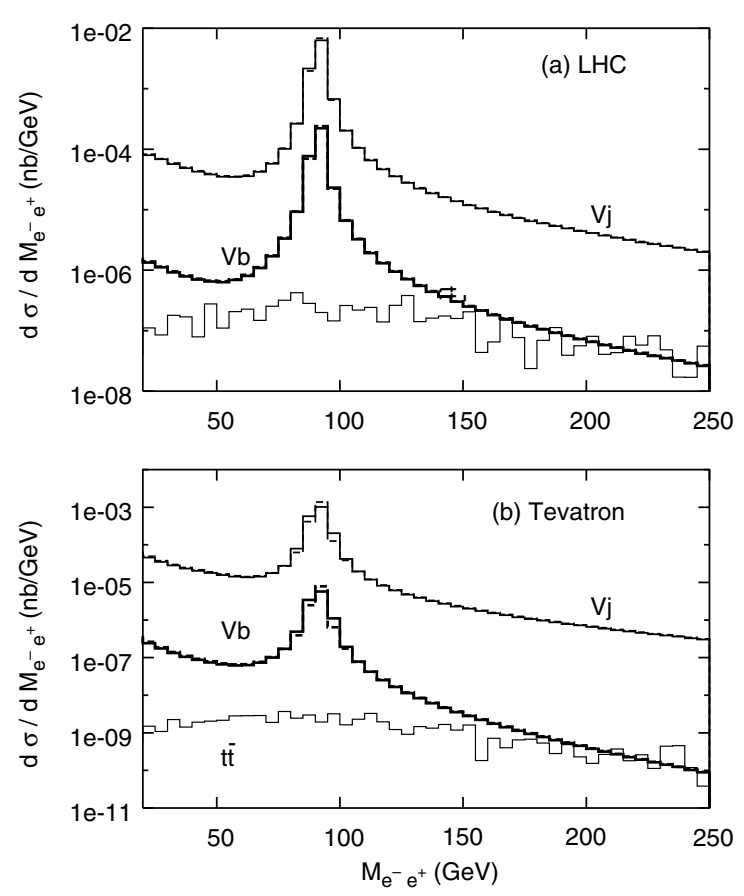

FIG. 1. Leading order $e^{-} e^{+} j(V j)$ and $e^{-} e^{+} \stackrel{(-)}{b}(V b$ and $t \bar{t})$ cross sections as functions of $M_{e^{-}} e^{+}$for the processes, cuts, and efficiencies discussed in the text at LHC (a) and Tevatron (b).

a factor of 30 smaller in this case, but the asymmetry is much larger because only $g \stackrel{(-)}{b}$ collisions contribute. As explained, NLO corrections are not included but they are eventually needed to describe the data. In Fig. 1 we also plot the top pair background, $p \stackrel{(-)}{p} \rightarrow t \bar{t} \rightarrow W^{+} W^{-} b \bar{b} \rightarrow$ $\nu_{e} \bar{\nu}_{e} e^{+} e^{-} b \bar{b}$ [15], and consider the case of losing one $b$. We assume the same $b$-tagging efficiency and that the second $b$ jet is missed if $p_{t}^{b}<50 \mathrm{GeV}$. We also require that the total transverse momentum $p_{t}<20 \mathrm{GeV}, p=$ $p^{e^{-}}+p^{e^{+}}+p^{b}$. The resulting distribution is rather flat and the smearing makes no difference. In the $M_{e^{-}} e^{+}$ interval between 75 and $105 \mathrm{GeV}$, the signal is 200 times larger, $\sigma^{V b}=1.7 \mathrm{pb}$, whereas $\sigma^{t \bar{t}}=0.008 \mathrm{pb}$. This background is further reduced by a factor of 1.25 if the $b$ jet is only missed for $p_{t}^{b}<20 \mathrm{GeV}$. So, we neglect it in the following. In any case, its mixed $e \mu$ decays can also provide a further handle on $t \bar{t}$. In Figs. 1 and 2(b) we plot the same cross sections and asymmetries but for Tevatron. At $2 \mathrm{TeV}$ the $q \bar{q}$ collisions dominate and the asymmetry adapted to these events $A_{\mathrm{FB}}$ is much larger. The applied smearing and cuts are given in Eqs. (5) and (6). In particular, for the $t \bar{t}$ background we mimic the missed $\stackrel{(-)}{b}$ by demanding $p_{t}^{b}<30 \mathrm{GeV}$ and also require $p_{t}<20 \mathrm{GeV}$. In such conditions, we find that the $V b$ signal is 700 times larger in the $M_{e^{-}} e^{+}$range between 75 and $105 \mathrm{GeV}, \sigma^{V b}=58 \mathrm{fb}$, whereas $\sigma^{t \bar{t}}=0.08 \mathrm{fb}$. Other $W \underset{(-)}{\text { pair }}$ backgrounds such as $p \stackrel{(-)}{p} \rightarrow$ $W^{+} W^{-} j, W^{+} W^{-} \stackrel{-(-)}{b}, \quad$ or $W^{+} W^{-} j j, W^{+} W^{-} b \bar{b} \quad$ with only one jet detected, which can be large a priori, can
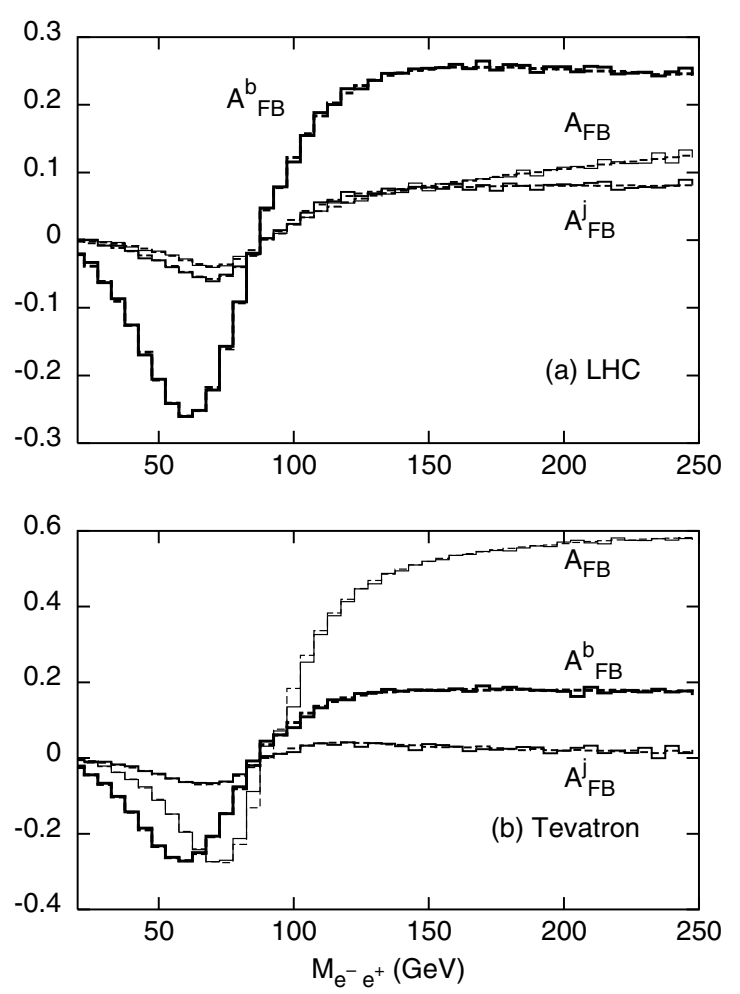

FIG. 2. Forward-backward electron asymmetries defined in the text as functions of $M_{e^{-}} e^{+}$for $e^{-} e^{+} j\left(A_{\mathrm{FB}}\right.$ and $\left.A_{\mathrm{FB}}^{j}\right)$ and $e^{-} e^{+} \stackrel{(-)}{b}\left(A_{\mathrm{FB}}^{b}\right)$ events in Fig. 1 at LHC (a) and Tevatron (b). The $t \bar{t}$ background is not included.

be further reduced, requiring small total transverse momentum.

Near the $Z$ pole, $M_{e^{-} e^{+}} \sim M_{Z}$, the asymmetries can be approximated by [3]

$$
A=b\left[a-\sin ^{2} \theta_{\mathrm{eff}}^{\text {lept }}\left(M_{Z}^{2}\right)\right],
$$

translating then their measurement into a precise determination of $\sin ^{2} \theta_{\text {eff }}^{\text {lept }}\left(M_{Z}^{2}\right)$. In Table I we collect the asymmetry estimates and their statistical precision, the corresponding $b$ and $a$ values in Eq. (7) and the precision reach $\delta \sin ^{2} \theta_{\text {eff }}^{\text {lept }}$ of LHC and Tevatron for $M_{e^{-}} e^{+}$in the range $[75,105] \mathrm{GeV}$ and two sets of cuts. The first set has been used throughout this Letter and is given in Eq. (6), whereas the second one requires a smaller minimum jet transverse momentum, $p_{t}^{j}>20(10) \mathrm{GeV}$ at LHC (Tevatron). These less stringent cuts increase the number of events and then improve the statistical precision by $10 \%$ to $50 \%$ depending on the asymmetry and collider. We have not tried to optimize them at this stage, but it will have to be done when dealing with real data and the experimental inefficiencies are known. The cross sections are also gathered in Table I. All the estimates include the smearing in Eq. (5). The results without smearing are very similar, except for the $A_{\mathrm{FB}}^{j}$ asymmetry and the second set of cuts for which $A_{\mathrm{FB}}^{j}$ is $20 \%$ smaller (larger) at LHC (Tevatron).

We have assumed throughout this Letter a $\stackrel{(-)}{b}$-tagging efficiency $\epsilon$ of $50 \%$. This is too optimistic, especially 
TABLE I. Estimates for the $e^{-} e^{+} j$ and $e^{-} e^{+} \stackrel{(-)}{b}$ cross sections and asymmetries defined in the text with $M_{e^{-}} e^{+}$in the range $[75,105] \mathrm{GeV}$. The integrated luminosity as well as the smearing, cuts, and tagging efficiency can be found in the text. The statistical precisions are also given, to be compared with the current effective weak mixing angle uncertainties at LEP and SLD from asymmetries only $1.7 \times 10^{-4}$, and from $A_{\mathrm{FB}}^{b}$ at the $Z$ pole $3.1 \times 10^{-4}$ [7].

\begin{tabular}{cccccccc}
\hline \hline & $\sigma(\mathrm{pb})$ & & $A$ & $\delta A$ & $b$ & $a$ & $\delta \sin ^{2} \theta_{\mathrm{eff}}^{\text {lept }}$ \\
\hline \multirow{2}{*}{$\mathrm{LHC}$} & $\sigma^{V j}=49$ & $A_{\mathrm{FB}}$ & $8.708 \times 10^{-3}$ & $4.5 \times 10^{-4}$ & 0.346 & 0.2491 & $1.3 \times 10^{-3}$ \\
$p_{t}^{j}>50 \mathrm{GeV}$ & & $A_{\mathrm{FB}}^{j}$ & $1.170 \times 10^{-2}$ & $4.5 \times 10^{-4}$ & 0.467 & 0.2490 & $9.7 \times 10^{-4}$ \\
& $\sigma^{V b}=1.7$ & $A_{\mathrm{FB}}^{b}$ & $7.136 \times 10^{-2}$ & $2.4 \times 10^{-3}$ & 2.723 & 0.2502 & $8.9 \times 10^{-4}$ \\
& $\sigma^{V j}=167$ & $A_{\mathrm{FB}}$ & $8.207 \times 10^{-3}$ & $2.4 \times 10^{-4}$ & 0.357 & 0.2469 & $6.9 \times 10^{-4}$ \\
$p_{t}^{j}>20 \mathrm{GeV}$ & & $A_{\mathrm{FB}}^{j}$ & $8.077 \times 10^{-3}$ & $2.4 \times 10^{-4}$ & 0.289 & 0.2519 & $8.5 \times 10^{-4}$ \\
& $\sigma^{V b}=5.9$ & $A_{\mathrm{FB}}^{b}$ & $5.667 \times 10^{-2}$ & $1.3 \times 10^{-3}$ & 2.187 & 0.2499 & $6.0 \times 10^{-4}$ \\
Tevatron & $\sigma^{V j}=9.7$ & $A_{\mathrm{FB}}$ & $5.944 \times 10^{-2}$ & $3.2 \times 10^{-3}$ & 2.658 & 0.2463 & $1.2 \times 10^{-3}$ \\
$p_{t}^{j}>30 \mathrm{GeV}$ & & $A_{\mathrm{FB}}^{j}$ & $8.306 \times 10^{-3}$ & $3.2 \times 10^{-3}$ & 0.386 & 0.2455 & $8.3 \times 10^{-3}$ \\
& $\sigma^{V b}=0.06$ & $A_{\mathrm{FB}}^{b}$ & $5.373 \times 10^{-2}$ & $4.2 \times 10^{-2}$ & 2.206 & 0.2483 & $1.9 \times 10^{-2}$ \\
& $\sigma^{V j}=39$ & $A_{\mathrm{FB}}$ & $6.722 \times 10^{-2}$ & $1.6 \times 10^{-3}$ & 3.005 & 0.2463 & $5.3 \times 10^{-4}$ \\
$p_{t}^{j}>10 \mathrm{GeV}$ & & $A_{\mathrm{FB}}^{j}$ & $6.374 \times 10^{-3}$ & $1.6 \times 10^{-3}$ & 0.357 & 0.2418 & $4.5 \times 10^{-3}$ \\
& $\sigma^{V b}=0.21$ & $A_{\mathrm{FB}}^{b}$ & $4.709 \times 10^{-2}$ & $2.2 \times 10^{-2}$ & 1.924 & 0.2484 & $1.1 \times 10^{-2}$ \\
\hline \hline
\end{tabular}

because we assume no contamination $\omega$, and, in particular, no charge misidentification. The statistical precisions $\delta A$ and $\delta \sin ^{2} \theta_{\text {eff }}^{\text {lept }}$ are proportional to $\epsilon^{-1 / 2}$, and the asymmetries $A$ and coefficients $b$ in Eq. (7) to $1-2 \omega$. This means, in particular, that the contamination multiplies $\delta \sin ^{2} \theta_{\text {eff }}^{\text {lept }}$ by $(1-2 \omega)^{-1}$. Hence, if we only consider semileptonic $b$ decays, implying $\epsilon \sim 0.1$ and $\omega \sim 0, \delta A$ and $\delta \sin ^{2} \theta_{\text {eff }}^{\text {lept }}$ increase by a factor $\sim 2$. In practice, we must try to maximize the quality factor $\mathrm{Q}=\epsilon(1-2 \omega)^{2}$ [16]. The statistical precisions given in Table I are certainly optimistic for systematic errors are also sizeable. To approach the quoted precisions will be an experimental challenge.

In summary, we have pointed out that the large $V j$ production cross section at hadron colliders and the possibility of measuring the lepton asymmetries relative to the final jet allow for a precise determination of the effective electroweak mixing angle. If there is an efficient $b$-tagging and charge identification, these events with a $b$ jet also allow for a new determination of $A_{\mathrm{FB}}^{b}$. The corresponding statistical precisions are collected in Table I. As in Drell-Yan production [17], this process is also sensitive to new physics for large $M_{e^{-}} e^{+}$, especially to new gauge bosons.

We thank J. A. Aguilar Saavedra, A. Bueno, R. Pittau, and J. Santiago for useful comments. This work was supported in part by MCYT under Contract No. FPA2000-1558, Junta de Andalucía group FQM 101 and the European Community's Human Potential Programme under Contract No. HPRN-CT-2000-00149 Physics at Colliders.

[1] R. Brock et al., in Proceedings of the Workshop on QCD and Weak Boson Physics in Run II, Batavia, 1999, edited by U. Baur, R. K. Ellis, and D. Zeppenfeld, p. 78 (FERMILAB-PUB-00/297, 2000).
[2] S. Haywood et al., in Proceedings of the Workshop on Standard Model Physics (and more) at the LHC, CERN, 1999, edited by G. Altarelli and M. L. Mangano, p. 117 (CERN 2000-004, 2000).

[3] J. L. Rosner, Phys. Lett. B 221, 85 (1989).

[4] P. Fischer, U. Becker, and J. Kirkby, Phys. Lett. B 356, 404 (1995).

[5] F. Abe et al., Phys. Rev. Lett. 77, 2616 (1996).

[6] U. Baur, S. Keller, and W. K. Sakumoto, Phys. Rev. D 57, 199 (1998); U. Baur, O. Brein, W. Hollik, C. Schappacher, and D. Wackeroth, Phys. Rev. D 65, 033007 (2002).

[7] LEP Collaborations, LEP Electroweak Working Group, and SLD Heavy Flavour and Electroweak Groups, CERN-EP/2001-098 [hep-ex/0112021].

[8] S. Catani and M. H. Seymour, Phys. Lett. B 378, 287 (1996); D. Dicus, T. Stelzer, Z. Sullivan, and S. Willenbrock, Phys. Rev. D 59, 094016 (1999).

[9] J. C. Collins and D. E. Soper, Phys. Rev. D 16, 2219 (1977).

[10] A. D. Martin, R. G. Roberts, W. J. Stirling, and R. S. Thorne, Eur. Phys. J. C 14, 133 (2000).

[11] R. Hamberg, W. L. van Neerven, and T. Matsuura, Nucl. Phys. B359, 343 (1991).

[12] A. Airapetian et al., ATLAS Detector and Physics Performance Technical Design Report, CERN-LHCC 99-14/15, http://atlasinfo.cern.ch/ATLAS/internal/tdr. html.

[13] G. L. Bayatian et al., CMS: the TriDAS Project Technical Design Report, CERN/LHCC 2000-38, http:// cmsdoc.cern.ch/cms/TDR/TRIGGER-public/trigger.html.

[14] F. Abe et al., Phys. Rev. D 45, 3921 (1992).

[15] R. Kleiss and W. J. Stirling, Z. Phys. C 40, 419 (1988).

[16] BABAR Collaboration, B. Aubert et al., SLAC-PUB9060 [hep-ex/0201020].

[17] F. del Aguila, M. Quirós, and F. Zwirner, Nucl. Phys. B287, 419 (1987); J. L. Rosner, Phys. Rev. D 54, 1078 (1996); T. Affolder et al., Phys. Rev. Lett. 87, 131802 (2001). 